\title{
THE GROTHENDIECK GROUP FOR STABLE HOMOTOPY IS FREE
}

\author{
BY PETER FREYD ${ }^{1}$
}

Communicated by E. Spanier, July 5, 1966

Let $H_{n}^{m}$ be the set of homotopy types of base-pointed finite complexes of dimension $\leqq m$ and connectivity $\geqq n$. We shall always assume that $2 n \geqq m$, in other words, that we are working in the "stable range".

$H_{n}^{m}$ is closed under the "wedge" operation ( $X \vee Y$ is obtained by identifying the base points in the disjoint union of $X$ and $Y$ ). Chang [1] has classified the wedge indecomposables in the case $m \leqq n+3$ and has shown that a unique wedge decomposition theorem holds in $H_{n}^{n+3}, n \geqq 3$.

Proposition 1. Unique wedge decomposition fails in $\boldsymbol{H}_{5}^{10}$. Indeed $\left(H_{5}^{10}, \vee\right)$ fails to be a cancellation semigroup. The same pathology holds for any $H_{n}^{m}, m \geqq n+5,2 n \geqq m$.

The easiest example: Let $\nu \in \pi_{9}\left(S^{6}\right)$ be a map of order 8. Let Cone $(\nu)$ be its mapping cone. Then $S^{6} \vee$ Cone $(\nu) \simeq S^{6} \vee$ Cone $(3 \nu)$ but Cone $(\nu)$ $\not$ Cone $(3 \nu)$. (The isomorphism uses only that 3 is prime to the order of $\nu$, the nonisomorphism uses only that 3 is not congruent to $\pm 1 \bmod$ the order of $\nu . \nu$ could not be of order $2,3,4$, or 6 . Hence a similar example is avoided in the range covered by Chang.)

Let $C_{n}^{m}$ be the cancellation semigroup obtained from $\left(H_{n}^{m}, V\right)$ by defining $X \equiv Y$ if there exists $Z$ such that $X \vee Z \simeq Y \vee Z$.

Theorem 2. $X \equiv Y$ iff for the bouquet of spheres, $B$, with the same Betti numbers as $X$ it is the case that $X \bigvee B \simeq Y \bigvee B$.

It follows that the inclusion $H_{n}^{m} \rightarrow H_{n}^{m+1}$ remains a monomorphism when we pass to $C_{n}^{m} \rightarrow C_{n}^{m+1}$. The suspension functor preserves wedges and hence we obtain a homomorphism from $\left(H_{n}^{m}, \bigvee\right)$ to $\left(H_{n+1}^{m+1}, \bigvee\right)$. By Freudenthal's theorem $H_{n}^{m} \rightarrow H_{n+1}^{m+1}$ is an isomorphism. We obtain a family of monomorphisms $C_{n}^{m} \rightarrow C_{n^{\prime}}^{n^{\prime}}, n \leqq n^{\prime}, m \leqq m^{\prime}$ the direct limit of which we'll call $S$. Each $C_{n}^{m}$ is a sub-semigroup of $S$ and it may be noted that each of the statements below about $S$ and its ambient group specializes nicely to $C_{n}^{m}$ and its ambient group.

1 This research was supported in part by grant GP 4252 from the National Science Foundation. 
Corollary 3. Given $\gamma \in \pi_{n}\left(S^{m}\right), n \neq m, \gamma \neq 0$ then $[$ Cone $(\gamma)]$ is indecomposable in $\mathbf{S}$.

Proposition 4. S is not free, i.e. it is not a unique factorization semigroup.

The easiest example. Let $\alpha \in \pi_{q}\left(S^{6}\right)$ be a map of order $3, \nu \in \pi_{9}\left(S^{6}\right)$ of order 8. Then Cone $(\alpha) \bigvee$ Cone $(\nu) \simeq S^{6} \bigvee S^{10} \bigvee$ Cone $(\alpha+\nu)$. The example lives in $H_{5}^{10}$. For any $\gamma, \delta \in \pi_{n}\left(S^{m}\right), n \neq m, \gamma, \delta$ of co-prime orders the same pathology may be exhibited.

The above example depends upon the mixing of prime integers. We may explicate that dependency by defining a space $X$ to be $p$ primary ( $p$ a prime integer) if there exist maps $f: B \rightarrow X, g: X \rightarrow B$, where $B$ is the bouquet of spheres with the same Betti numbers as $X$, such that $g f=p^{n} \cdot 1_{B}$, some $n$.

Spheres are $p$-primary for any $p$. The only spaces which are $p$ primary for more than one $p$ are bouquets of spheres.

Let $S_{p}$ be the cancellation semigroup obtained by restricting attention to $p$-primary spaces.

THEOREM 5. $S_{p}$ is free, i.e. a unique factorization semigroup. Moreover $[X]$ is indecomposable in $S_{p}$ iff $X$ is wedge indecomposable.

(It is known that a space in the stable range is wedge indecomposable iff its only idempotent endomorphisms are 0 and 1 [2].)

Let $G$ be $S$ made in to a group (the Grothendieck group for stable homotopy). Let $B: G \rightarrow G$ be the map which sends $[X]$ to the bouquet of spheres $\left[B_{X}\right]$ with the same Betti numbers as $X . B$ is idempotent. Let $G_{S}$ be its image, $G^{*}$ its kernel. $G=G_{S} \oplus G^{*}$. Note that $G_{S}$ is clearly freely generated by the spheres. Let $G_{p}^{*}$ be the subgroup of $G^{*}$ generated by elements of the form $[X]-\left[B_{X}\right]$ where $X$ is $p$ primary. Note that $S_{p}$ made into a group is $G_{S} \oplus G_{p}^{*}$. Hence $G_{p}^{*}$ is free.

Theorem 6. $G^{*}$ is the internal direct sum of the $G_{p}^{* \prime} s . G$ is free. It is freely generated by the set $\left\{S^{n} \mid S^{n}\right.$ an n-sphere $\} \cup\left\{[X]-\left[B_{x}\right] \mid X\right.$ a wedge indecomposable primary space $\}$.

The next was a contention of Milnor.

THEOREM 7. $[X]-[Y]$ has zero component in $G_{s} \oplus G_{p}^{*}$ iff $X$ and $Y$ have the same Betti numbers and there exists $f: X \rightarrow Y$ such that $H_{*}\left(f ; Z_{p}\right)$ is an isomorphism where $\boldsymbol{Z}_{p}$ can be interpreted either as the prime field or the p-adic integers.

CoROLlaRy 8. With the smash product as multiplication, $G_{p}^{*}$ is an ideal. 
The proofs rely heavily upon the representation of the stable homotopy category $\&$ (of which $G$ is the Grothendieck group) as the full subcategory of projectives in a Frobenius category $\mathcal{F}$ [3]. (A Frobenius category is an Abelian category in which projectives and injectives coincide and in which there are enough of them in both senses.) Statements 1 through 4 require repeated use of the Schanuel lemma applied in $F$. Theorem 5 depends upon a suitable modification of the Nakayama lemma. Theorem 6 uses the Schanuel lemma to represent the Grothendieck group in another more easily handled Grothendieck group arising from $F$. For Theorem 7 it is necessary to localize $\mathfrak{F}$ by factoring out, a la Gabriel, the Serre class of objects whose identity maps are of finite order prime to $p$. This $p$-localization of $\mathcal{F}$ has many nice properties: it is Frobenius; its indecomposable injectives are spaces and they are absolutely indecomposable, i.e. are essential extensions of every nontrivial subobject; each of its objects has an injective envelope; it is self-dual and hence each of its objects has a projective co-envelope.

We obtain an almost-answer to the question which inaugurated the investigation: can mapping cones in the stable range be identified by their homotopy properties?

THEOREM 9. If

$$
X \stackrel{f}{\longrightarrow} Y \longrightarrow Z \longrightarrow S X \stackrel{S f}{\longrightarrow} S Y
$$

is such that an exact sequence of abelian groups results whenever a corepresentable functor is applied (or if preferred, whenever any cohomology theory is applied) then $[Z]$ is equal to $[\mathrm{Cone}(f)]$ in the Grothendieck group, i.e. $Z \equiv$ Cone $(f)$.

There does exist a sequence

$$
S^{9} \stackrel{\nu}{\longrightarrow} S^{6} \longrightarrow \text { Cone }(3 v) \longrightarrow S^{10} \stackrel{S \nu}{\longrightarrow} S^{7}
$$

satisfying the hypothesis. Hence $Z$ need not be of the same homotopy type as Cone $(f)$.

\section{BIBLIOGRAPHY}

1. S. C. Chang, Homotopy invariants and continuous mappings, Proc. Roy. Soc. Ser. A, 202 (1950), 253-263.

2. P. Freyd, Splitting homotopy idempotents, Proceedings of the Conference on Categorical Algebra, Springer-Verlag, 1966 (to appear).

3. - Stable homotopy, Proceedings of the Conference on Categorical Algebra, Springer-Verlag, 1966 (to appear).

University of Pennsylvania 